\title{
Diagnóstico, manejo y actualización en cuerpo extraño aerodigestivo
}

\section{Diagnosis, treatment and update in foreign body of the aerodigestive tract}

\author{
Cristián Lara $\mathrm{M}^{1}$, Gabriel Faba $\mathrm{C}^{1}$, Jorge Caro $\mathrm{L}^{1}$.
}

\begin{abstract}
RESUMEN
日 cuerpo extraño de la vía aerodigestiva es una patología que sigue siendo un reto para el otorrinolaringólogo. Son cerca de 3.000 muertes al año en EUU. por asfixia asociado a cuerpo extraño y la incidencia se ha mantenido en los últimos años. $\theta$ grupo de los niños sigue siendo el mayor riesgo.

Con el objetivo de establecer una revisión del tema se realizó una búsqueda en bases de datos y OVID con las palabras claves de esophagic foreign body, foreign body airway, bronchoscopy. Se seleccionaron referencias entre 1997 y 2007 que permitieran además entregar una puesta al día en epidemiología, clínica, diagnóstico y tratamiento.

Key Words: Querpo extraño vía aérea, broncoscopía, cuerpo extraño esofágico.
\end{abstract}

\begin{abstract}
Foreign body in the aerial and digestive pathway is a challenging pathology for the ORL specialist. It results in about 3000 deaths per year in USA, with a persisting incidence in recent years. Children persist as the higher risk group.

With the aim of reviewing the literature on this subject, a data base and OVID search was performed, using "esophagic foreign body", "foreign body airway" and "bronchoscopy" as key words. References published between 1997 and 2007 were selected, which in addition allows for an epidemiologic, clinical, diagnosis and treatment update.
\end{abstract}

Key words. Foreign body, pediatric airway, esophagus.

1 Médico Otorrinolaringólogo del Departamento de Otorrinolaringología de la Pontificia Universidad Católica de Chile. 


\section{INTRODUCCIÓN}

Se define como cuerpo extraño (CE) de la vía aerodigestiva a la presencia de cualquier objeto tanto en la vía aérea (desde la nariz hasta el árbol bronquial) como en la hipofaringe y esófago. Sin lugar a dudas el manejo de esta situación es un gran reto para el otorrinolaringólogo ${ }^{1}$. De los CE vía aerodigestiva sólo $40 \%$ de ellos, aproximadamente, pasarán a la vía aérea.

El cuerpo extraño aspirado (CEA) de vía aérea (VA) es una situación grave y no poco común, es más frecuente en los niños, aunque también se ve en grupos de adultos mayores. Debido al compromiso agudo de la VA y sus complicaciones -que pueden ir desde la tos persistente hasta la muerte del paciente ${ }^{2}$ - es que se considera una urgencia que requiere de la inmediata intervención.

La presencia de un CE nasal es un cuadro frecuente en los servicios de urgencias, pero de una importancia menor. Produce obstrucción nasal, estornudos, rinorrea mucosa o mucopurulenta, habitualmente unilateral, que más tardíamente puede presentar hasta mal olor. Rara vez produce dolor. La extracción debe ser con la máxima premura para evitar la migración del CE y la aspiración del mismo¹. Principal preocupación se debe tener en el caso de las pilas, las que reaccionan rápidamente con la secreción y la mucosa nasal provocando en minutos una quemadura de la misma e incluso comprometer hasta el cartílago septal.

En el caso de los CE de hipofaringe también es un cuadro clínico que se ve más en los servicios de urgencias. El paciente refiere tener la sensación de cuerpo extraño en la hipofaringe. Habitualmente son CE punzantes -espinas de pescados, huesos de pollos quebrados, etc.-y que por las características de la fase faríngea de la deglución se impactan en la vallécula e incluso pueden llegar al seno piriforme. El tratamiento es la pronta extracción para evitar la migración a la VAy hacia espacios profundos del cuello con las consecuentes complicaciones. Se describen casos de extracción de CE de la hipofaringe con pinza Magill.

Otra situación frecuente es la presencia esofágica de un $\mathrm{CE}$, al que le dedicaremos especial atención más adelante.

Pero sin duda que el CE de la vía aérea baja es una constante preocupación y un reto para el $\mathrm{ORL}$, y será el tema principal de nuestra revisión.

\section{EPIDEMIOLOGÍA}

En EE.UU. ocurren más de 3.000 muertes al año que se asocian a asfixia por un $\mathrm{CEA}^{3}$, la incidencia de CEA se ha mantenido en el tiempo, teniendo al grupo de menores de 15 años como el grupo de riesgo. Pero es en el subgrupo de los niños entre 1 a 3 años donde se concentran los casos ${ }^{1-7}$, teniendo una especial incidencia en el grupo de los 2 años, que según algunas series publicadas pueden alcanzar hasta el $48 \%$ del total de casos de muerte accidentales en población pediátrica ${ }^{4}$.

En el grupo de los adultos se encuentran dos peak de incidencia: en la década de los 60 y otro en la década de los 80 , siendo el primero más importante que el segundo.

En España la mortalidad alcanza el 0,9\% en global, pero no se debe olvidar que la asfixia por CEA representa el $40 \%$ de las muertes accidentales en menores de un año ${ }^{1}$. En México describen mortalidad al año de $0,26 \%$ a $5 \%$ en población pediátrica por CEA.

En Chile, la situación es similar. En 1985 fue una de las tres causas de muerte más importante en ORL, con 1,7 de las muertes por cada 100.000 hab.; para 1995, fueron algo más de 700 muertes por CEA en la población pediátrica ${ }^{8}$. En la actualidad en nuestro país la tasa promedio de mortalidad por CEA en patología otorrinolaringológica entre los años 1991 y 1999 es de 4,99 por 100.000 habitantes, correspondiendo al $68 \%$ de las muertes de causas otorrinolaringológicas. El $40 \%$ de estas muertes ocurre en población menor de un año, que alcanza una tasa de mortalidad de 90,5 por 100.000 nacidos vivos. Luego entre el año de vida y hasta los 14 años la mortalidad disminuye a 0,8 por 100.000. En lo que respecta a la tasa de mortalidad, en población general, por esta causa de muerte existe una disminución para el periodo de años mencionado, pasando de una tasa de 7,85 por 100.000 habitantes en 1991 a una de 2,54 por 100.00 habitantes, lo que equivale a una disminución del $68 \%$ de la mortalidad por esta causa en Chile, según una revisión nacional ${ }^{9}$.

\section{FISIOPATOLOGÍA}

El CEA es más frecuente en los niños por distintos motivos, que a su vez explican los peak de inciden- 
cia en los distintos años de vida. Primero, la inmadurez neurológica propia del desarrollo del niño, que se expresa en una deglución y función glótica inmadura. Segundo, se debe considerar la carencia de molares, lo que dificulta el adecuado manejo de los alimentos y por tanto aumenta el riesgo de aspiración. Luego, otro factor importante es el interés a esta edad por explorar su entorno a través de la boca, a esto se suma que al mismo tiempo que lo hacen juegan y corren ${ }^{3,6,10}$. En los niños mayores se puede deber a una respiración bucal concomitante a tener en la boca elementos de uso cotidiano y se asocia a juegos más bruscos 0 intensos. Mientras que en los adultos se debe principalmente a: desórdenes 0 alteración de la deglución, a trastornos 0 enfermedades neurológicas o neuromusculares ${ }^{4} \mathrm{y}$ al uso de prótesis dentales, entre otras.

Una vez que el CEA pasa a la VA y toca la laringe, se produce un brusco cierre de ésta. Esto corresponde al espasmo laríngeo que puede provocar la dificultad respiratoria, ahogo y hasta cianosis. Además se producirá una intensa tos, que puede llegar a ser hematizante, para eliminar el CEA, que si no es eficaz en eliminarlo, entonces, se debe apoyar con maniobras médicas inmediatas para evitar la irreversibilidad del cuadro'. Lo anterior se conoce como síndrome de Penetración ${ }^{9}$. Luego, existe una segunda fase, en que el CEA ya pasó a subglotis y que puede durar de minutos hasta meses e incluso puede ser asintomática. Lo anterior dependerá del grado de obstrucción que estará dado por el tamaño del CEA y su localización. Una tercera fase corresponde a las complicaciones que el CEA genere: inflamación, inflamación-infección, entre otras. Aquí se debe tener en cuenta la confusión con otros diagnósticos diferenciales.

\section{CLÍNICA}

La clínica irá muy de la mano de la fisiopatología y obviamente estará condicionada por el tamaño del CEA, de su localización y de su composición ${ }^{1,3,4}$.

Según algunas series dentro de los signos y síntomas del CEA, el más frecuente es el síndrome de penetración que puede llegar a estar en el $49 \%$ de los $\operatorname{casos}^{4,6,7,11-14}$, otros síntomas frecuentes son la tos persistente, fiebre, disnea, sibilancias ${ }^{4,7,10}$.

Es importante entender además que la clínica va a depender de la localización del CEA, así podremos encontrar: cuerpo extraño laríngeo, traqueal y bronquial.

a. Cuerpo extraño laríngeo. Es la localización menos frecuente en las series, va entre el $2 \%$ a $12 \%$. Si el tamaño del CEA es grande provocará la obstrucción parcial o total de la glotis provocando: dificultad respiratoria, disnea, cianosis e incluso la muerte. Mientras que si el CEA es más pequeño provocará: estridor respiratorio, disfonía 0 afonía, tos crupal, odinofagia ${ }^{1}$. Los síntomas los causa el propio CEA y/o la inflamación que provoca en la VA.

b. Cuerpo extraño traqueal. Es una localización más frecuente que la anterior, corresponde alrededor del $7 \%$ de los casos. Los síntomas más habituales son: la tos persistente, estridor y sofocación. Se describe un signo característico del CEA, que es el choque del mismo en la tráquea 0 un golpe audible del CEA, mientras el paciente ventila ${ }^{1}$.

c. Cuerpo extraño bronquial. Es la localización más frecuente del CEA, llegando a alcanzar el $80 \%$ de los casos, con un claro predominio del bronquio derecho $0^{1,3,6,8,11,12}$. En esta ubicación los síntomas más frecuentes son la tos y las sibilancias. Pero dependiendo de la fase en la que se haga el diagnóstico los síntomas pueden variar. En función del tamaño y del grado de obstrucción podremos encontrar desde leves sibilancias a crisis de asma ${ }^{5}$ (paso bidireccional del flujo), enfisema (paso unidireccional del flujo) y hasta atelectasias (obstrucción total) o incluso simular una neumonía u otras patologías pulmonares.

Para el caso de los CE bronquiales se describe una triada que consiste en: tos, sibilancias y disminución de ruidos respiratorios unilaterales.

Pero si el diagnóstico no lo hacemos a tiempo, también es posible encontrar síntomas propios de las complicaciones del CEA, que se describirán más adelantes.

\section{DIAGNÓSTICO}

El diagnóstico se basa en tener una alta sospecha clínica ${ }^{1,16}$, en la historia clínica, en el examen físico y en las pruebas de apoyo.

a. Historia clínica. La historia clínica completa podría tener hasta $80 \%$ de sensibilidad según algu- 
nos autores ${ }^{1}$. Es más, distintos autores afirman que la historia clínica es la clave del diagnóstico en el CEA ${ }^{3-5,7,715,16}$. Es importante para el médico tener siempre presente además, que el CEA puede imitar otras patologías pulmonares.

Es importante interrogar dirigidamente a los acompañantes, pues cuando el hecho ha sido presenciado por terceros el diagnóstico ofrece menos dudas ${ }^{32,35}$. En ocasiones cuando el evento no es presenciado, entonces la dificultad es mayor e incluso el interrogatorio dirigido no aportará para el diagnostico definitivo. Pero, si la historia no es sugerente de CEA o no fue presenciada por terceros, tendremos que sospecharla fuertemente ${ }^{12}$ frente a la presencia de: disnea de inicio súbito y/o tos paroxística en un niño de 2 ó 3 años de edad. Si la tos toma un aspecto de croup, entonces el inicio brusco de disfonía 0 afonía es importante también.

Otros elementos del interrogatorio serán el buscar dirigidamente por antecedentes de neumonías, atelectasias a repetición, más aún si es del mismo lóbulo o segmento. Sibilancias o tos de difícil y arrastrado manejo.

b. Examen físico. Otro elemento importante será el examen físico del paciente. Ya hemos mencionado los signos que debemos buscar en nuestro paciente, pero es importante señalar que desde $15 \%$ a $40 \%$ de los casos el examen físico puede ser totalmente normal ${ }^{1,3}$. En el servicio de urgencia rara vez nos enfrentaremos al niño con claros signos de insuficiencia respiratoria aguda obstructiva o con signos de asfixia.

A la observación se podría evidenciar una disminución de la movilidad de uno de los hemitórax, 0 francamente dificultad y esfuerzo respiratorio. La auscultación pulmonar será un elemento crítico con una alta sensibilidad pero de baja especificidad'. Se debe buscar a la auscultación: signos de obstrucción como sibilancias, disminución del murmullo pulmonar o la ausencia de éste ${ }^{6,7}$. La sibilancia en niños no asmáticos y en ausencia de respuesta a tratamiento adecuado debe plantear la sospecha de un CEA.

Otras situaciones que deben hacer sospechar de un CEA son: neumonías a repetición, más aún si afectan al mismo segmento; tos intratable, atelectasias, hiperinsuflación, abscesos pulmonares, entre otras. c. Imagenología. Frente a un paciente con sospecha de CEA y aun cuando sabemos que tiene una menor sensibilidad, se debe plantear el uso de la radiografía de cuello y tórax anteroposterior y lateral. Lo anterior no sólo nos puede apoyar en el diagnóstico de CEA, sino que nos permitirá en ocasiones, diferenciar si el CE está en vía aérea 0 esófago. Por otro lado el CEA detectable en la radiografía convencional alcanza sólo entre el $4 \%$ al 11\% 1,4,7,11,13,16 según diferentes estudios. Diversos estudios entre los años 1998 y 2004 señalan que la radiografía convencional tiene una sensibilidad sólo del $27 \%$ al $80 \%$ y una especificidad entre el $67 \%$ al $85 \%{ }^{11}$. Esto depende mucho del tipo de CEA.

Teniendo presente lo anterior el hallazgo más típico en el niño es la hiperinsuflación (49\%), luego le siguen la atelectasia, neumonía, el CEA visible y una radiografía normal $(14 \%, 13 \%$, $4 \%$ y $12 \%$, respectivamente). Mientras que en el adulto la atelectasia es el hallazgo más importante con $50 \%$ de los casos. Le siguen la neumonía, hiperinsuflación, el cuerpo extraño visible y una radiografía normal con $17 \%, 17 \%$, $11 \%, 11 \%$, respectivamente 4 .

La tomografía computada (TC) también puede ser un apoyo en el diagnóstico del CEA, se describe una sensibilidad de $100 \%$ y una especificidad del $90 \%$. Además en la TC helicoidal con reconstrucción de la vía aérea se pueden realizar las denominadas endoscopías virtuales, en las que se realiza un recorrido virtual 3D por el interior de la vía aérea. En algunas series reportan hasta $100 \%$ de sensibilidad y especificidad al compararla con la broncoscopía rígida, mientras que la especificidad y sensibilidad de la radiografía simple con la broncoscopía rígida fue de $85 \%$ y $68 \%$, respectivamente ${ }^{11}$.

Frente a un paciente con sospecha de CEA y con una radiografía negativa, se ha planteado el uso de la TC 0 de endoscopia rígida de inmediato; ambas posiciones mejoran el proceso diagnóstico pero lo encarecen. Es por eso y lo descrito anteriormente es que hay grupos que sugieren de entrada el uso de la TC para el diagnóstico de CEA, pues le consideran una estrategia diagnóstica de mayor beneficio para el paciente y más costo-efectiva. En el caso de no tener posibilidad de una TC, entonces consideran de 
inmediato la endoscopía rígida ${ }^{17}$. Además la TC permite la evaluación de la densidad del parénquima pulmonar. En los CEA vegetales la especificidad y sensibilidad puede disminuir ${ }^{11}$. En un interesante estudio de niños con sospecha de CE aspirado, se les realizó una evaluación clínica, radiológica y de laboratorio, pre broncoscopía. De éstos en $76 \%$ el juicio clínico para realizar la broncoscopía estaba correcto, y encontraron que la hiperinsuflación local (imagenología), recuento de glóbulos blancos elevados (laboratorio) y la crisis de ahogo se asociaron fuertemente a una broncoscopía positiva para CEA. De manera que concluyen que una broncoscopía está fuertemente recomendada en presencia de al menos 2 de los 3 elementos descritos ${ }^{14,15}$.

\section{TIPOS DE CEA}

La mayoría de Ios CEA son de origen orgáni$\mathrm{CO}^{2,6,7,13,16,17}$, tanto en el grupo de los niños como en los adultos ( $91 \%$ y $59 \%$, respectivamente). Más de la mitad de los CEA orgánicos en el grupo de los niños fueron maní y en el grupo de los adultos los alimentos son un grupo importante. Los CEA no orgánicos en los niños son más habitualmente plásticos y trozos de juguetes, mientras que en los adultos se describen dientes y restos de dentadura.

\section{RETRASO DEL DIAGNÓSTICO}

En virtud de lo anterior se debe tener en cuenta que frente a un paciente con un CEA el diagnóstico puede llegar a ser fácil o complejo 0 incluso no realizado. Algunas series describen un retraso en el diagnóstico de CEA de entre horas a meses ${ }^{2,6,7}$. En el grupo de los niños el diagnóstico se demora menos que en los adultos y en el grupo de menores de 1 año el diagnóstico se hace en mucho menos tiempo, no hay relación entre el retraso del diagnostico ni con la localización del CEA ni con los síntomas. En una revisión de 1.887 broncoscopías de pacientes con sospecha de CEA, tan solo $43 \%$ de los pacientes ingresaron antes de las primeras 24 horas y hubo 4,3\% que lo hizo después de un mes $^{7}$.

\section{DIAGNÓSTICO DIFERENCIAL}

Se debe diferenciar de un crup laríngeo, epiglotitis, disfonías o afonías agudas, cuando está en laringe. A nivel traqueobronquial se debe diferenciar de asma 0 crisis asmáticas, malformaciones, broncomalacia, tos psicógena, neumonías, abscesos retrofaríngeos, entre otras. Importante es tener en cuenta que cuadros respiratorios de evolución tórpida y arrastrada se debe plantear al menos la sospecha del CEA ${ }^{3,6}$.

\section{TRATAMIENTO}

El manejo podrá ser de dos tipos, médico y quirúrgico.

a. Manejo médico. El manejo médico queda reservado para aquel paciente que está en una crisis aguda y súbita de insuficiencia respiratoria por el CEA y que requiere una asistencia e intervención inmediata. Clásicamente se describen: golpes en la espalda, golpe-compresión torácica y la maniobra de Heimlich ${ }^{1}$. La elección de una de ellas depende de la edad del paciente.

Si la obstrucción es completa y el niño tiene menos de un año se pone al lactante decúbito prono, con la cabeza más abajo que el tronco y apoyándole con los dedos la mandíbula, tratando de mantener la cabeza ligeramente extendida; se golpea moderadamente y con rapidez con el talón de la mano en zona interescapular 5 veces. Luego se pone en la misma ubicación pero decúbito dorsal, sosteniendo la cabeza con la mano y siempre más abajo que el tronco y se harán 5 compresiones rápidas y moderadamente fuerte. Se revisará la boca y se extraerá el CEA, si no se ve el CEA, se repite la maniobra.

El fracaso de la segunda repetición debe obligarnos a dar respiración boca a boca, o con $\mathrm{AMBU}^{\circledR}$ y a la intubación (recordar que el tubo puede llevar el CAE más a distal y/o quedar obstruyendo el tubo, se recomienda una vez permeabilizado la vía aérea revisar y/o cambiar el tubo).

En la obstrucción completa de un niño que tiene más de un año se puede realizar las maniobras de compresión torácica, se recomienda la maniobra de Heimlich, el fracaso de ella debe generar una cascada de reacción igual a la anterior. 
Si la obstrucción es incompleta, el periodo crítico ha pasado y se debe estabilizar el paciente para la extracción definitiva del CEA, mientras se debe mantener en control permanente del paciente para evitar una nueva crisis ${ }^{1}$.

b. Manejo quirúrgico. El manejo quirúrgico lo desarrollaremos más intenso en el siguiente punto.

\section{MANEJO QUIRÚRGICO}

A pesar de lo descrito anteriormente el manejo del CEA es frecuentemente quirúrgico. Se deben tener en cuenta al menos los siguientes elementos: el equipo médico, el equipamiento, el procedimiento anestésico y el procedimiento de remoción del CEA, lo que discutiremos a continuación.

a. Equipo médico. El retiro del CEA es un gran reto para el equipo. Se considera una emergencia y requiere la inmediata intervención ${ }^{4}$. En lugares donde no es frecuente la extracción de un CEA puede generar gran stress en el grupo y una falta de coordinación entre los mismos.

El manejo del CEA se debe trabajar en equipo con mucha antelación ${ }^{2}$ y previa coordinación, de manera que al momento de la extracción del mismo los roles de cada uno de los miembros del equipo sea la correcta y no entorpezca el procedimiento que en momentos se puede tornar altamente laborioso y dificultoso.

Se debe considerar y coordinar a los distintos actores entre ellos: la unidad de cuidados intensiva pediátrica 0 adulta según corresponda, que se debe interiorizar de las posibles complicaciones de la vía aérea en estos casos, al manejo y el apoyo vital del paciente con vía aérea difícil. Además será importante la incor- poración de un médico broncopulmonar (pediátrico 0 adulto, según corresponda). EI médico otorrinolaringólogo, que debe ser un endoscopista con experiencia en la vía aérea pediátrica y habituado a los equipos y al manejo de ellos. También es fundamental un médico anestesiólogo entrenado en el manejo de la vía aérea pediátrica compleja ${ }^{2,3}$. Todos deben actuar coordinadamente en el momento de la emergencia, de manera que conocer el trabajo y los quehaceres de cada uno, previamente, es indispensable. Debiera ser nuestra preocupación que lo anterior se cumpla.

b. Equipamiento. El equipo endoscópico debe ser el adecuado para cada edad y paciente, de lo contrario el paciente se expone a un aumento del traumatismo y edema de la vía aérea durante la extracción del CEA (Tabla 1), de manera que debe ser revisado y seleccionado por el endoscopista antes de iniciar el procedimiento ${ }^{3}$. El equipo debe tener: laringoscopios, que se utilizarán para extraer CE laríngeos o para facilitar el paso del broncoscopio, y 2 sets de broncoscopios, en virtud de que alguno falle 3 . Deben de incorporarse múltiples tipos de forceps con acción pasiva y activa para la extracción de los diversos tipos CEA que podamos encontrar. Se prefiere que éstos cuenten ópticas que permitirán la magnificación del CEA, por lo tanto su mejor identificación y permanente control sobre ellos ${ }^{3}$. El uso de cámara en las ópticas permite que todo el equipo en pabellón esté visualizando el procedimiento. Además se deben contar con las apropiadas aspiraciones tanto flexibles como rígidas para el desarrollo de la extracción del CEA4.

c. Procedimiento anestésico. El procedimiento debe ser altamente coordinado entre anestesista y otorrinolaringólogo, se recomienda el repaso de

Tabla 1, Selección de broncoscopio y laringoscopio por edad

\begin{tabular}{|lcc|}
\hline Edad & Tamaño broncoscopio (diámetro externo en mm) & Tamaño laringoscopio \\
\hline Prematuro & $2,5(3,7)$ & 8 \\
RN de término (hasta 3 meses) & $3(4,8)$ & 8 \\
6 meses (3 a 18 meses) & $3,5(5,7)$ & 9 \\
18 meses (1 a 3 años) & $3,7(6,3)$ & $9,5-11$ \\
3 años (2 a 6 años) & $4(6,7)$ & $9,5-12$ \\
7 años (5 a 10 años) & $5(7,8)$ & 12 \\
10 años y más & $6(8,2)$ & 16 \\
\hline
\end{tabular}


las acciones previo al ingreso a pabellón entre ellos ${ }^{3}$.

La extracción del CEA debe ser bajo anestesia general| ${ }^{1,3-5,8,17)}$. Se recomienda régimen cero por boca de 6 a 8 horas para alimentos y de 2 a 4 horas para líquidos livianos. En caso de urgencia se trata como tal.

Se debe tener en cuenta el tipo de inducción anestésica y sus posibles complicaciones. La inducción endovenosa puede hacer perder el control de la vía aérea mientras que la inducción inhalatoria podría generar aspiración 0 impactar el CEA en la vía aérea ${ }^{8}$.

El anestesista tendrá que ventilar al paciente por el broncoscopio, manteniendo buenos niveles de oxigenación y adecuada ventilación. Para ellos hay grupos que durante la extracción del CEA pediátrico utilizan la ventilación espontánea. Por el contrario, otros grupos que durante la anestesia de la extracción del CEA mantienen el control de la ventilación. Aun cuando ambas técnicas anestésicas son apropiadas y efectivas en el tratamiento de la extracción del $\mathrm{CEA}^{2}$, dependerán del paciente. Por otro lado, otros grupos concluyen que no es posible mantener una adecuada profundidad de la anestesia con ventilación espontánea para la extracción del CEA con broncoscopio rígido, por lo que proponen el control de la ventilación por parte del anestesista ${ }^{17}$. El anestesista y el médico tratante debe definir la mejor decisión. Se recomienda también el uso de lidocaína local en las cuerdas vocales ${ }^{3}$ (con atomizador, al $10 \%$, uso tópico). Además se pueden usar relajantes musculares en el justo momento y previo a la introducción del broncoscopio. Como es obvio, se debe además mantener la monitorización constante del paciente con saturometría, presión arterial, monitorización del ritmo cardiaco, monitoreo del CO2, entre otras. La anestesia será entregada a través del broncoscopio ${ }^{17}$.

El manejo anestésico es de exclusiva reponsabilidad del anestesista, pero lo anterior es necesario que lo conozca el otorrinolaringólogo, pues tendrá que discutir el procedimiento y ajustar detalles del mismo con el anestesista previo al inicio de la extracción del CEA, como ya se había comentado.

d. Procedimiento de extracción del CEA: La extracción del CEA es por broncoscopía rígida 3,5,7,12,13,15,17.

\section{BRONCOSCOPIA RÍGIDA (BR)}

La BR la describe G. Killian en 1897, persistiendo casi como se describió desde entonces; el mismo Killian describe años después, la primera extracción de un cuerpo extraño desde un bronquio. Jackson continúa el desarrollo de la BR, durante el siglo pasado. Además los avances tecnológicos completan este desarrollo de la BR con los lentes, endoscopios y pinzas -broncoscopía rígida moderna- teniendo en la actualidad un gran uso diagnóstico y terapéutico.

El procedimiento de elección para la extracción del CEA es la BR, aun cuando hay publicaciones en que se ha realizado extracción de CEA con broncoscopía flexible en niños, esto no es lo habitual ${ }^{19}$ pues el broncoscopio flexible se introduce retirando la mascarilla laríngea, perdiendo el control de la vía aérea, con una ventilación y oxigenación menos eficiente, con una escasa diversidad de pinzas y fórceps para extracción de CEA y con una peor resolución de imagen al compararle con el telescopio ${ }^{19}$. Por otro lado, el flexible ocupa la totalidad de la vía aérea pediátrica, lo que más aún nos deja en una desmejorada situación tanto para el otorrinolaringólogo como para el anestesista. Por tanto, la BR entrega una morbilidad y mortalidad más baja y adecuada en lo que respecta al riesgo y control de la vía aérea del niño ${ }^{12}$.

El broncoscopio flexible tiene utilidad frente a un CEA en el diagnóstico y podría utilizarse en adultos ${ }^{2}$, pero la recomendación es siempre el uso del broncoscopio rígido por lo ya descrito. Además la broncoscopía flexible tiene como contraindicación relativa le hipoxemia y está contraindicada en el CEA de vía aérea pediátrica ${ }^{20}$.

\section{CUIDADOS POSPROCEDIMIENTOS}

La broncoscopía rígida no está exenta de complicaciones, entre ellas tenemos: obstrucción de la vía aérea, neumotórax-neumomediastino, hemoptisis, edemas y laceraciones laríngeas y de la mucosa de tráquea y bronquios, estenosis, fístula traqueoesofágica y la no extracción del CEA, entre otras. Es importante entonces que se monitorice estos elementos, posterior a la extracción en sí.

Es necesario un posoperado de al menos 4 horas. El uso de antibióticos sólo en el caso que 
veamos pus en la extracción. La adrenalina racémica y corticoides se usan si tenemos sospecha de edema, dificultad del paso por laringe 0 inapropiado tamaño de broncoscopio. El seguimiento con radiografía de tórax no es de rigor y también sólo frente a la sospecha de alguna situación especial en la extracción del CEA. La necesidad de la UCI se evalúa entre el ORL y el anestesista, no siendo de rigor en caso de fácil extracción $n^{5}$.

\section{COMPLICACIONES DEL CEA}

Contrario a lo que se cree, que la BR es un procedimiento simple en pediatría, se describen serias complicaciones aun en manos experimentadas ${ }^{11}$.

Se han descrito variadas complicaciones inherentes principalmente al diagnóstico tardío del CEA. En una revisión de 1.269 niños con CEA aerodigestivo, el $15 \%$ de ellos se trató por más de 7 días por asma, neumonías o fiebre atribuida a otitis media aguda, sin pensar en el CEA, a pesar de el antecedente de tos y ahogo. De estos niños, 61 se complicaron con patología pulmonar, prolongando y complejizando su hospitalización (entre ellos: neumonía y atelectasias como las más comunes) ${ }^{13}$.

Otras complicaciones descritas fueron: no poder extraer el CEA, sangrados (hemoptisis) por granulaciones o rupturas de vasos circundantes al CEA. Neumomediastino y neumotórax más rara vez. También se describen más rara vez abscesos y otras infecciones mayores ${ }^{1,3,13,18}$ y la necesidad de traqueostomía en algunas ocasiones.

Importante es tener presente que dentro de los 1.269 niños, 30 llegaron en paro respiratorio, de ellos 3 fallecieron y 22 se recuperaron sin secuelas ${ }^{13}$.

En el intraoperatorio es importante evaluar la ventilación del paciente, si existen dificultades inmediatamente revise su equipo, con el anestesista asegure que el sistema de flujo de aire sea óptimo, desde el paciente hasta la máquina de anestesia. De lo contrario piense en la intubación endotraqueal y en complicaciones como neumotórax, entre otras, y su manejo.

Es importante coordinar con el equipo de cirugía de tórax por posibles complicaciones intraoperatorias como el sangrado incoercible de los bronquios, lo mismo si el cuerpo extraño está ubicado muy distal. En la literatura se describe que en la extracción de CEA por BR se puede requerir una toracotomía en 2,5\% $\%^{2,7}$.

En la vía aérea pediátrica la extracción de cuerpo extraño es mucho más dificultoso y más riesgoso que en la del adulto.

\section{CUERPO EXTRAÑO ESOFÁGICO (CEE)}

EI CEE es una situación de relativa frecuencia y que principalmente se ve en población pediátrica ${ }^{22,24}$. Se pueden impactar en el esófago pero también pueden presentarse impactados en otros sitios del tracto digestivo ${ }^{22}$.

En cuanto a la epidemiología del problema, en EE.UU. son cerca de 1.000 niños al año que se les extrae un $\mathrm{CEE}^{24}$. Es más frecuente en población pediátrica ( $80 \%$ de los CEE), que en la adulta. De éstos es más frecuente antes de los 5 años y son de sexo masculino principalmente ${ }^{23}$. La moneda es el CEE más frecuente en niños, le siguen elementos de plásticos y partes de juguetes, mientras que en población adulta son alimentos -hueso de pollo 0 espina de pescado- principalmente ${ }^{25-27}$. Aun cuando han aumentado los "pins" que en algunas series muestran porcentajes similares a las monedas.

La causa de los CEE es similar a la de los CEA en vía aérea, y es básicamente a la curiosidad del niño de explorar el mundo con su boca.

En lo que respecta a la ubicación es importante mencionar que el $5 \%$ de los CE pueden quedar pegados en la orofringe, $80 \%$ se pueden ubicar en el estómago, 10\% puede pasar al intestino y es sólo el $20 \%$ que se va a ubicar en el esófago. De ellos el $90 \%$ a 80\% pasará con tratamiento médico y/u observación (sale por deposición), el 10\% a 20\% requerirá extracción por esofagoscopía y sólo el $1 \%$ requerirá cirugía ${ }^{25}$.

El CEE tienden a impactarse más en el tercio superior, esófago cervical, a nivel del músculo cricofaríngeo. Le siguen de lejos el tercio medio y el tercio inferior, respectivamente.

El cuadro clínico se caracteriza por el antecedente de la deglución del CEE, que muchas veces no es claro 0 no está presente. El inicio del cuadro se puede tardar varias horas en iniciarse. Niños muy pequeños incluso pueden no dar síntomas o confundir con otro cuadro por la presencia de tos, en ocasiones asociado a estridor y anorexia. Lo más clásico es que se presente con sialorrea y disfagia 
(ambas cercanas al 70\% en distintas series) ${ }^{23}, 1$ de cada 4 pacientes van a presentar también vómitos 0 regurgitación, náuseas estará presente en $15 \%$ y el dolor cervical y retroesternal en $18 \%$ y $4 \%$, respectivamente, entre los más importantes. Recordar que algunos niños pueden ser asintomáticos y sólo puede ser un hallazgo de un estudio radiológico ${ }^{23}$.

Para el diagnóstico es importante la sospecha clínica ${ }^{24}$ y estudios de apoyo como la radiografía simple de cuello y tórax AP y lateral, como la mayor parte de los cuerpos extraños son monedas en niños se podrá pesquisar.

También se describe el uso de la TC que se puede requerir cuando el CEE no se encuentra en la radiografía simple y existe una alta sospecha, o en las complicaciones del CEE. Pero previamente se debe evaluar el esofagograma con medio de contraste.

El tratamiento es la extracción del CEE. Este se realiza con esofagoscopía rígida (ER) la que tiene un éxito de entre el $95 \%$ a $100 \%$, según la serie. Frente al fracaso de este procedimiento requerirá de tratamiento con cirugía. La ER tiene la ventaja de mantener la vía aérea protegida, se pueden extraer distintos tipos de CEE, existe la visualización directa del CEE y del estado del esófago. La extracción del CEE, al igual que el CEA de VA, debe ser extraído en block con el esofagoscopio rígido.

Otras formas de extraer CEE es a través de endoscopía digestiva alta flexible, la que sólo en algunos CEE puede ser útil, pero si se decide sedar al menor, entonces se debe considerar a la ER. Otras formas descritas son la extracción con catéter con balón distal fluoroscópico (o sonda Folley), que pueden ser más fácil y de bajo costo, pero más insegura pues es un procedimiento a ciegas ${ }^{26}$. También se describe el uso del fórceps de Magill bajo visión directa 0 guiada endoscópicamente $23,24,28,29$.

La resolución quirúrgica queda para el fracaso de la extracción endoscópica ${ }^{26}$ y de acuerdo al nivel en que se encuentre el CEE se puede realizar por vía cervical 0 torácica.

Las complicaciones pueden ir desde el no encontrar el CEE hasta complicaciones como perforación esofágica, mediastinitis, neumomediastino, abscesos, estenosis esofágica, fístulas traqueoesofágicas, compromiso de tejidos blandos ${ }^{24}$, incluso se describe migración y fístulas a pie ${ }^{30}$ (que puede hacer sospechar de un proceso más crónico), entre otras. También se pueden presentar hemorragias y hematemesis se- cundarias. Especial énfasis se debe hacer a las pilas tipo botón que rápidamente pueden provocar complicaciones, por tanto urge su extracción ${ }^{24}$.

Los cuidados posoperatorios tendrá que decidirlos el endocospista en virtud de las características del procedimiento y pensando en las posibles complicaciones descritas.

\section{PREVENCIÓN}

Probablemente sea la línea trascendental en el manejo del CEA de vía aérea y el CEE. Una buena intervención en los padres, guarderías y responsables de las mismas pueda ser útil y así como leyes en la industria de juguetes y otros elementos a los que nuestros niños tengan acces $0^{1}$.

\section{BIBLIOGRAFÍA}

1. Korta J, Alberdi A, Belloso, J. Cuerpos extraños en la vía respiratoria. Protocolos de la asociación española de pediatría, capitulo 7. Consultado el 20 de mayo de 2007. Disponible en www.aeped.es/protocolos

2. Soysal 0 , Kuzucu A, Ulutas H. Tracheobronchial foreign body aspiration: a continuing challenge. Aolaryngol Head Neck Surg 2006; 135(2): 223-6.

3. Murray A, Walner D. Methods in instrumentation for removal of airway foreign bodies. Operative techniques in otolaryngology, 2002; 13: 2-5.

4. Baharloo F, Veyckemans F, Francis C, Biettlot MP, RODENSTEIN DO. Tracheobronchial foreign bodies: presentation and management in children and adults. Chest 1999; 115(5): 1357-62.

5. MurRay A. Foreign Bodies of the airway. Consultado Mayo de 2007. Disponible en www.emedicine.com

6. Skoulakis CE, Doxas PG, Papadakis CE, Proimos E, Christodoulou P, Bizakis JG, Velegrakis GA, Mamoulakis D, Helidonis ES. Bronchoscopy for foreign body removal in children. A review and analysis of 210 cases. Int $J$ Pediatr Otorhinolaryngol 2000; 53(2): 143-8.

7. Aydogan lB, Tuncer U, Soylu L, Kiroglu M, Ozsahinoglu C. Rigid bronchoscopy for the suspicion of foreign body in the airway. Int $J$ Pediatr Otorhinolaryngol 2006; 70(5): 823-8. 
8. Lemus R, Fernández M. Anestesia en cuerpos extraños de via aerea y esófago. Tres años de experiencia. Rev Aorrinolaringol Or Cabeza Quello 1999; 59: 73-8.

9. Béjar M, Cevo J, Romero I, IñIguez SR. Mortalidad nacional en otorrinolaringología. Rev Atorrinolaringol Oir Cabeza Ouello 2007; 67: 31-7.

10. Prado F, Yánez J, Boza M, Herrera P, Guillén B, Hernández $\mathrm{H}$, Quezada G. Obstrucción de la vía aérea por cuerpo extraño. Manejo endoscópico combinado. Rev Chil Pediatr v.70 n.5 Santiago set. 1999.

11. Haliloglu M, Ciftci A0, Оto A, Gumus B, Tanyel FC, Senocak Me, Buyukpamukcu N, Besim A. CT virtual bronchoscopy in the evaluation of children with suspected foreign body aspiration. Eur J Radiol 2003; 48(2): 188-92.

12. Latifi $X$, Mustafa $A$, Hysenas $Q$. Rigid tracheobronchoscopy in the management of airway foreign bodies: 10 years experience in Kosovo. Int J Pediatr Aorhinolaryngol 2006; 70(12): 2055-9.

13. Reilly J, Thompson J, Macarthur C, Pransky S, Beste D, Smith M, Gray S, Manning S, Walter M, Derkay C, Muntz H, Friedman E, Myer CM, Seibert R, Riding K, Cuyler J, Todd W, Smith R. Pediatric aerodigestive foreign body injuries are complications related to timeliness of diagnosis. Laryngoscope 1997; 107(1): 17-20.

14. Heyer CM, Bollmeier ME, Rossler L, Nuesslein TG, Stephan V, Bauer TT, Rieger CH. Evaluation of clinical, radiologic, and laboratory prebronchoscopy findings in children with suspected foreign body aspiration. J Pediatr Surg 2006; 41(11): 1882-8.

15. Even L, Heno N, Talmon Y, Samet E, Zonis Z, Kugelman A. Diagnostic evaluation of foreign body aspiration in children: a prospective study. J Pediatr Surg 2005; 40(7): 1122-7.

16. Ibrahim Sersar S, Hamza UA, Abdelhameed WA, Abulmaaty RA, Gowael IN, Moussa SA, Almorsi SM, HafEz MM. Inhaled foreign bodies: management according to early or late presentation. Eur $J$ Cardiothorac Surg 2005; 28(3): 369-74.

17. Soodan A, Pawar D, Subramanium R. Anesthesia for removal of inhaled foreign bodies in children.

Pediatric Anesthesia 2004; 14: 947-52.

18. Shrime M, Johnson P, Stewart M. Cost-Effective Diagnosis of Ingested Foreign Bodies. Laryngoscope 2007; 117: 785-93.

19. Flores S, García R, Núñez C. Extracción de cuerpos extraños de la vía aérea en niños mediante broncoscopía flexible. Rev inst nal enf resp mex 2005; (18)2: 103-8.

20. F Midulla, J de Blic, A Barbato, A Bushz, E Eber, $S$ Kotechaf, E Haxby, C Moretti, P Pohunek, $\mathrm{F}$ RATJenzz. Flexible endoscopy of paediatric airways. Eur Respir J 2003; 22: 698-708.

21. Loré J, Medina J. An atlas of Head and Neck Surgery, $4^{\circ}$ Ed., 2005.

22. Myers E. Operative Otolaryngology. Head and Neck Surgery 1997; Vol. I: 558-74.

23. Mahazza T, Batieha A, Suboh M, Khrais T. Esophageal foreign bodies: a Jordanian experience. Int $J$ Pediatr Corhinolaryngol 2002; 64: 225-7.

24. Yalcin S, Karnak I, Ciftci A, Emin M, Cahit F, BüYÜKPAMUKCU N. Foreign body ingestion in children: an analysis of pediatric surgical practice. Pediatr Surg Int 2007; 23: 755-61.

25. Rooks V, Chung E. Esophagus, Foreign Body. Consultado Agosto de 2007. Disponible en www.emedicine.com

26. Maluenda C, Varea V. Ingesta de cuerpos extrños. Consultado en Agosto de 2007. Disponible en www.aeped.es/protocolos

27. Benito J, Del Cubillo A, Porras A. Cuerpos extraños esofágicos: nuestra experiencia en diez años. Acta Otorrinolaringol Esp 2003; 54: 281-5.

28. Peña A. Extracción de cuerpos extraño de hipofaringe por medio de pinza de MacGill. Rev Corrinolaringol Or Cabeza Quello 2002; 62: 303.

29. Maseda E, Ablanedo C, Fernández MJ. Migración y fistulaización a través de la piel de cuerpo extraño faríngeo (espina de pescado). Acta Otorrinolaringol Esp 2006; 57; 474-6.

\author{
Dirección: Dr. Cristián Lara M \\ Departamento de Otorrinolaringología, Pontificia Universidad Católica de Chile \\ Marcoleta $352,2^{\circ}$ Piso. Santiago, Chile. \\ E mail: cristianlaram@gmail.com
}

